\title{
Understanding Heterogeneous Deformation in Polycrystalline Al 6061 by in Situ SEM Deformation and HREBSD Characterization
}

\author{
Jeremy Yoo ${ }^{1}$, Jay Carroll ${ }^{2}$, John Emery ${ }^{2}$ and Josh Kacher ${ }^{1}$ \\ ${ }^{1 .}$ Georgia Institute of Technology, Materials Science and Engineering, Georgia, USA \\ 2. Sandia National Laboratories, New Mexico, USA
}

Al 6061 is an important structural alloy useful for its excellent strength-to-weight ratio and corrosion resistance. Its microstructure is composed of a complex array of precipitates as well as the heterogeneities implicitly associated with polycrystalline materials. This complex microstructure results in deformation processes occurring across length scales in a heterogeneous matter. As such, it is important to understand how local processes interact synergistically to develop mesoscale strain heterogeneities that ultimately lead to failure.

One such tool capable of mapping out the deformation evolution over large areas is electron backscatter diffraction (EBSD). The original work by Adams et al. showed that, using a Nye's tensor formulation coupled with Kroner's analysis, EBSD was capable of quantitatively mapping out dislocation densities [1]. Later work has shown that the minimum resolvable dislocation density of this approach $\left(\rho_{\min }^{G N D}\right)$ is approximately related to the step size at which the data are collected $(L)$ and the angular resolution of the characterization technique $(\theta)$ according to [2]:

$\rho_{\min }^{G N D}=\frac{\theta_{\min }}{L b}$

Where $b$ is the Burgers vector of the dislocations in the system. Accordingly, $\rho_{\min }^{G N D}$ can be decreased either by increasing L, sacrificing spatial resolution in the process, or improving $\theta$. One approach to improving $\theta$ is by high angular resolution EBSD (HREBSD), first viably demonstrated by Angus et al. and since adopted by a number of groups [3, 4]. This approach relates small shifts measured using cross-correlation functions in EBSD patterns to elastic strains and crystal rotations, improving the angular resolution of traditional EBSD by approximately two orders of magnitude. However, this approach comes at the cost of time, as it is reliant on the acquisition of high-quality EBSD patterns.

In this study, a multiscale EBSD approach was coupled with in situ scanning electron microscopy (SEM) deformation to tie together local deformation processes and mesoscale heterogeneities at interrupted stages of the deformation. A notched $0.8 \mathrm{~mm}$ thick Al 6061 sample was deformed to $2 \%$ strain with EBSD scans collected at $0 \%, 1 \%$, and $2 \%$ at a strain rate of $0.01 \% \mathrm{~s}^{-1}$. Large scans $(400 \times 400 \mu \mathrm{m}$ at $700 \mathrm{~nm}$ step size) were collected around the notch tip using traditional EBSD and smaller scans $(20 \times 20 \mu \mathrm{m}$ at $200 \mathrm{~nm}$ step size) were collected from regions of interest such as triple junctions. The dislocation density from both scans was approximated by $\rho^{G N D}=\frac{2 K A M}{L b}$ where KAM is the first nearest neighbor kernel average misorientation.

Figure 1 shows the deformation progression in the large scale scans. As can be seen, the deformation is concentrated in two lobes around the notch as well as a highly deformed region emitting from the center of the notch. The inset shows the high level of dislocation accumulation surrounding intermetallics contained in a grain ahead of the notch. 
The HREBSD scans, shown in Figure 2, display the dislocation evolution surrounding a triple junction. At $2 \%$ strain, a new rotation boundary formed with a misorientation angle across the boundary reaching a maximum of $20^{\circ}$ (located in the lower right corner of the image). Current work is focused on relating these deformation mechanisms to grain boundary character, crystal orientation, and precipitate distribution.

This work was supported by the Laboratory Directed Research and Development program at Sandia National Laboratories, a multi-program laboratory managed and operated by Sandia Corporation, a wholly owned subsidiary of Lockheed Martin Corporation, for the U.S. Department of Energy's National Nuclear Security Administration under contract DE-AC04-94AL85000.

\section{References:}

[1] Sun, S., B.L. Adams, and W.E. King, Phil. Mag. A 80 (2000), p. 9.

[2] Adams, B.L. and J. Kacher, Computers, Materials, \& Continua 14(3) (2009), p. 185.

[3] Wilkinson, A.J., G. Meaden, and D.J. Dingley, Ultramicroscopy 106 (2006), p. 307.

[4] Kacher, J. et al, Ultramicroscopy 109 (2009), p. 1148.

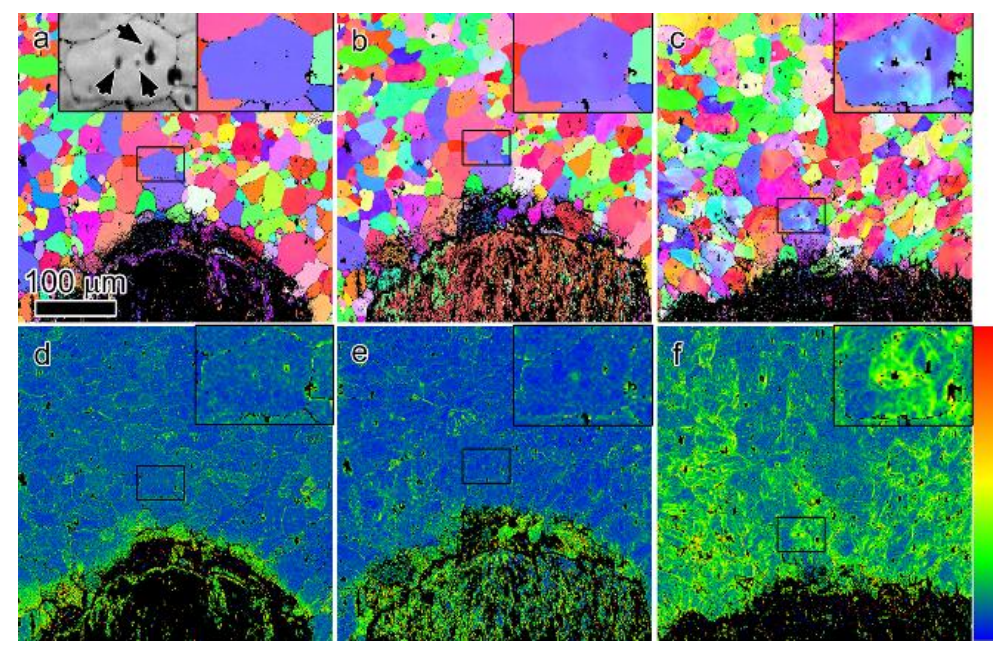

Figure 1. Large scale deformation map showing IPF map (a-c) and KAM map (d-f) at 0\% (a,d) $1 \%$ (b,e) and $2 \%(\mathrm{c}, \mathrm{f})$ deformation. a) includes inset of image quality map with intermetallics indicated. Scale bar relates to KAM maps and goes from 0 to $1 \times 10^{15} \mathrm{~m}^{-2}$.
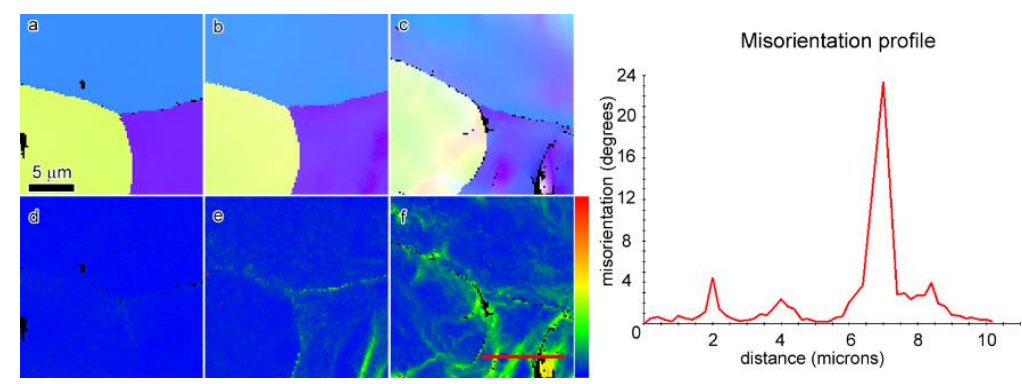

Figure 2. High-resolution deformation map showing IPF map (a-c) and KAM map (d-f) at 0\% (a,d) $1 \%$ $(\mathrm{b}, \mathrm{e})$ and $2 \%(\mathrm{c}, \mathrm{f})$ deformation. Scale bar relates to KAM maps and goes from 0 to $3.5 \times 10^{15} \mathrm{~m}^{-2}$. Misorientation profile is taken from the red line shown in (f). 